\title{
Effects of mexiletine, a CYP1A2 inhibitor, on tizanidine pharmacokinetics and pharmacodynamics
}

Kenji Momo, $\mathrm{MSc}{ }^{1}$, Masato Homma, $\mathrm{PhD}^{1^{*}}$, Yoshiko Osaka, MD, $\mathrm{PhD}{ }^{2}$, Shin-ichi Inomata, MD, $\mathrm{PhD}^{2}$, Makoto Tanaka, $\mathrm{MD}, \mathrm{PhD}^{2}$, Yukinao Kohda, $\mathrm{PhD}^{1}$

${ }^{1}$ Department of Pharmaceutical Sciences, Graduate School of Comprehensive Human Sciences, University of Tsukuba, Ibaraki, Japan

${ }^{2}$ Department of Anaesthesiology, Graduate School of Comprehensive Human Sciences, University of Tsukuba, Ibaraki, Japan

* Correspondence

Masato Homma, Department of Pharmaceutical Sciences, Graduate School of Comprehensive Human Sciences, University of Tsukuba, Ten-nodai 1-1-1, Tsukuba, Ibaraki 305-8575, Japan

Tel.: +81-29-853-3859

Fax: $+81-29-853-7025$

E-mail address: masatoh@md.tsukuba.ac.jp

Keywords: tizanidine; drug interaction; CYP1A2; mexiletine; blood concentration 


\section{ABSTRACT}

The aim of our study is to determine whether mexiletine, a CYP1A2 inhibitor, altered the pharmacokinetics and pharmacodynamics of tizanidine. Pharmacokinetics of tizanidine was examined in an open-label study in 12 healthy subjects after a single dose of tizanidine (2 mg) with and without mexiletine coadministration (50 mg, 3 times as a pre-treatment for a day and 2 times on the study day). Compared with tizanidine alone, mexiletine coadministration increased the peak plasma concentration $(1.8 \pm 0.8$ vs. $5.3 \pm 1.8 \mathrm{ng} / \mathrm{mL})$, area under the curve $(4.5 \pm 2.2$ vs. $15.4 \pm 6.5 \mathrm{ng} \bullet \mathrm{h} / \mathrm{mL})$ and the half-life $(1.3 \pm 0.2$ vs. $1.8 \pm 0.7 \mathrm{~h})$ of tizanidine, respectively $(P<0.05)$. Reduction in systolic blood pressure $(-10 \pm 8$ vs. $-24 \pm 7 \mathrm{mmHg})$ and diastolic blood pressure $(-10 \pm$ 7 vs. $-18 \pm 8 \mathrm{mmHg}$ ) after tizanidine administration were also significantly enhanced by coadministration of mexiletine $(P<0.01)$. Of the 15 patients treated with tizanidine and mexiletine, 4 suffered tizanidine-induced adverse effects such as drowsiness and dry mouth in the retrospective survey. Present results suggested that coadministration of mexiletine increased blood tizanidine concentrations and enhanced tizanidine pharmacodynamics in terms of reduction in blood pressure and adverse symptoms. 


\section{INTRODUCTION}

Tizanidine, an antispastic agent, is a sensitive substrate for cytochrome P450 (CYP) 1A2 as compared to theophylline and caffeine. ${ }^{1-3}$ Recent studies revealed that coadministration of potent CYP1A2 inhibitors such as ciprofloxacin and fluvoxamine with tizanidine induced the adverse effects of tizanidine (hypotension, low heart rate (HR), anuresis, drowsiness and speech disorders) accompanied with marked elevation in plasma tizanidine concentration. ${ }^{4-7}$ These combination therapies have been identified as contraindications in Japan. ${ }^{6-8}$

Mexiletine, a class 1b antiarrhythmic agent, is known to be a potent CYP1A2 inhibitor with a smaller inhibition constant as compared to ciprofloxacin in vitro. ${ }^{9,}{ }^{10}$ It has also been reported that coadministration of mexiletine increased plasma theophylline concentration via CYP1A2 inhibition in patients and healthy subjects. ${ }^{11-15}$ These findings suggest that mexiletine may alter tizanidine pharmacokinetics when both drugs are administered simultaneously.

This combination is sometimes used for the treatment of painful neuropathies. Although the drug interaction between mexiletine and tizanidine is stated in package inserts of pharmaceutical products of tizanidine hydrochloride, ${ }^{8}$ the magnitude and clinical effect of this interaction is unclear. To clarify this issue, we investigated the 
effects of mexiletine coadministration on the pharmacokinetics and pharmacodynamics of tizanidine in healthy subjects. Adverse effects were also surveyed retrospectively in the medical records of patients treated with this combination therapy.

\section{MATERIALS AND METHODS}

\section{Subjects}

Twelve healthy male subjects (29 $\pm 5 \mathrm{y} ; 69.9 \pm 12.1 \mathrm{~kg}$ ) including 5 smokers participated in the study (Table 1). We did not exclude smokers whose CYP1A2 activities may have potentially been induced, ${ }^{16}$ because we would like to examine the effects of smoking on the drug interaction in subgroup analyses (smokers and non-smokers). The subjects were considered healthy by their medical history, physical examination and routine laboratory tests before participating in the study. For safety reasons, subjects with systolic blood pressure (SBP) less than $110 \mathrm{mmHg}$ and/or CYP1A2 gene mutation $(3113 \mathrm{G} \rightarrow \mathrm{A})$ were excluded from the study. None of the subjects received medication at least 2 weeks prior to the study.

\section{Study design}

An open-label study with washout periods of $>5$ days was performed. Subjects ingested 2 mg tizanidine (Ternelin ${ }^{\circledR}$ Novartis Pharma, Basel, Switzerland) at 09:00 
(control phase) on the study day. After the washout period, the subjects ingested $50 \mathrm{mg}$ mexiletine hydrochloride (Mexitil ${ }^{\circledR}$ capsule, Nippon Boehringer Ingelheim, Tokyo, Japan; 3 times a day; at 08:00, 12:00 and 20:00) as pre-treatment. For safety reasons, pre-treatment with mexiletine was limited for one day. On the study day, subjects ingested $50 \mathrm{mg}$ mexiletine 2 times a day; at 08:00 and 12:00 and $2 \mathrm{mg}$ tizanidine at 09:00 (mexiletine phase). A low-fat meal was served at 07:00 and 12:00 as breakfast and lunch, respectively, on the study day. Grapefruit juice and alcohol and/or caffeine-containing beverages were not allowed during the study, 7 days and 1 day prior to the study, respectively.

Venous blood samples for determining plasma concentrations of tizanidine and mexiletine were collected immediately before and $0.5,1,2,3$ and $5 \mathrm{~h}$ after tizanidine administration. Urine sample was collected $8 \mathrm{~h}$ after tizanidine dosing. Urine and plasma separated from each whole blood sample was stored at $20^{\circ} \mathrm{C}$ until analys es. SBP, diastolic blood pressure (DBP) and HR were measured immediately after each blood sampling, for up to $5 \mathrm{~h}$ after tizanidine administration. An automatic oscillometric blood pressure monitor (HEM-1000; Omron Healthcare, Kyoto, Japan) was used for these measurements.

The study protocol was approved by the Ethics Committee of the University of 
Tsukuba, (Tsukuba, Japan). Written informed consent was obtained from all subjects.

\section{Determination of drug concentrations in plasma and urine}

Plasma and urine concentrations of tizanidine were determined using an API 3000 liquid chromatography-tandem mass spectrometry system (Applied Biosystems, Tokyo, Japan) according to the method of Nirogi et al with minor modification. ${ }^{17}$ Briefly, to 300 $\mu \mathrm{L}$ of plasma or urine, $25 \mu \mathrm{L}$ of internal standard (IS) (tamsulosin, 10 or $50 \mathrm{ng} / \mathrm{mL}$, Toronto Research Chemicals, North York, Canada) and $30 \mu \mathrm{L}$ of sodium hydroxide solution $(0.1 \%)$ was added and vortexed for 30 s. To extract tizanidine and IS, diethyl ether/dichloromethane $(7 / 3, \mathrm{v} / \mathrm{v})$ was vortexed with the mixture for $3 \mathrm{~min}$. The organic layer was evaporated to dryness under a stream of nitrogen. The dried extract was reconstituted with $100 \mu \mathrm{L}$ of mobile phase and the aliquot $(20 \mu \mathrm{L})$ injected into the chromatograph. The samples were separated on a $\mathrm{C}_{8}$ column (Inertsil C8, 4.6mm i.d. $\times 150 \mathrm{~mm}$, GL Sciences, Tokyo, Japan) using high-performance liquid chromatography (HPLC; LC-10AD VP system, Shimadzu, Kyoto, Japan). The analytical column was maintained at $40^{\circ} \mathrm{C}$. The HPLC mobile phase consisted of 10 $\mathrm{mM}$ ammonium formate and methanol (10:90, v/v), and the flow rate was set at $1.2 \mathrm{~mL} /$ min. Multiple reaction monitoring was used for mass spectrometric detection. Positive ionization mode was used in a heated nebulizer interface operating with the 
nebulizer-gas temperature set at $350^{\circ} \mathrm{C}$. The monitored $\mathrm{m} / \mathrm{z}$ for tizanidine and IS were 254.0/44.0 and 409.0/228.0, respectively. The ranges for tizanidine quantification in plasma and urine were $0.1-10 \mathrm{ng} / \mathrm{mL}$ and $0.5-50 \mathrm{ng} / \mathrm{mL}$, respectively. Corresponding within-day coefficient of variations (CVs) of tizanidine assay were $4.3-5.1 \%$ for plasma and $1.5-9.8 \%$ for urine.

The plasma concentrations of mexiletine were determined using HPLC (LC-10AT VP system, Shimadzu) in Mitsubishi Chemical Medience (Tokyo, Japan). The methods are described in the previous report by Mastropaolo et al. ${ }^{18}$ The lower limit of quantification was $0.1 \mu \mathrm{g} / \mathrm{mL}$ and within-day CVs for $0.1-2.0 \mu \mathrm{g} / \mathrm{mL}$ were $1.5-6.7 \%$.

All chemicals used for the assays were of analytical reagent grade.

\section{Pharmacokinetic and pharmacodynamic analyses}

Pharmacokinetic parameters such as peak concentration in plasma $\left(\mathrm{C}_{\max }\right)$, time to $\mathrm{C}_{\max }\left(\mathrm{t}_{\max }\right)$ and elimination half-life $\left(\mathrm{t}_{1 / 2}\right)$ of tizanidine were calculated from plasma concentration-time data. Area under the plasma concentration time curve (AUC) was calculated by the trapezoidal method. Apparent oral clearance (CL/F) was calculated as dose/AUC ${ }_{0-5}$. Non-renal clearance $\left(\mathrm{CL}_{\mathrm{NR}} / \mathrm{F}\right)$ was calculated as the difference between $\mathrm{CL} / \mathrm{F}$ and Renal clearance $\left(\mathrm{CL}_{\mathrm{R}} / \mathrm{F}\right)$. The pharmacokinetics of mexiletine was characterized by $\mathrm{C}_{\max }$ and AUC from 0 to $6 \mathrm{~h}$ after the morning dose of mexiletine in 
study day. A part of plasma mexiletine concentrations within the detector limit were predicted with a Bayesian estimate using Higuchi’s Bayesian program, PEDA VB ver. 1.0.0.58. ${ }^{19}$

Area under the effects (SBP, DBP and HR) versus time curves after administration of tizanidine were assessed as the pharmacodynamic marker for tizanidine.

\section{Genotyping of CYP1A2}

Genomic DNA was isolated from peripheral blood using the QIAamp DNA Mini Kit (Qiagen GmbH, Hilden, Germany). The genotyping of CYP1A2 at the position of $-3113 \mathrm{G} \rightarrow \mathrm{A}$ was conducted by polymerase chain reaction-restriction fragment length polymorphism according to the method of Chen et al. $^{20}$ This single nucleotide polymorphism is associated with low CYP1A2 activity.

\section{Retrospective survey}

We investigated medical records obtained from 1,563 patients treated with tizanidine in Tsukuba University Hospital and found 15 patients who received mexiletine simultaneously. The episodes associated with tizanidine administration (e.g. low blood pressure, low HR, drowsiness, anuresis and dry mouth), dosage, coadministration periods for tizanidine and mexiletine and patient characteristics (e.g. age, sex, body weight, disease, laboratory data and smoking status) were recorded. 


\section{Statistical analyses}

Data are expressed as percentages, ranges, mean \pm SD or 95\% CI. Pharmacokinetic and pharmacodynamic data between control and mexiletine phases in healthy subjects were compared by paired Student's t-test. The plasma concentration-time profiles of tizanidine and blood pressure-time profiles were compared by repeated-measures ANOVA. Comparing the patients' blood pressures with and without coadministration of tizanidine and mexiletine was conducted by paired Student's t-test. Difference was considered statistically significant when $P$ value was less than 0.05 .

\section{RESULTS}

\section{Pharmacokinetics of tizanidine and mexiletine}

The pharmacokinetic profile and parameters of tizanidine are compared between control and mexiletine phase in Fig. 1 and Table 2. Plasma tizanidine concentrations for mexiletine phase were significantly higher than control. Mean $\mathrm{AUC}_{0-5}$ and $\mathrm{C}_{\max }$ of tizanidine for the mexiletine phase were 3.6-fold and 3.1-fold higher than control (Table 2). The mean $t_{1 / 2}$ of tizanidine for the mexiletine phase was prolonged by 1.4 -fold as compared to control (Table 2). The $\mathrm{CL} / \mathrm{F}$ and $\mathrm{CL}_{\mathrm{NR}} / \mathrm{F}$ of tizanidine for the mexiletine phase decreased to one-third of control, although $\mathrm{CL}_{\mathrm{R}} / \mathrm{F}$ remained unchanged (Table 2). 
The pharmacokinetic parameters of tizanidine were compared between smokers and non-smokers. No significant difference in any parameters either for control and mexiletine phase was observed between two groups (data not shown).

Plasma mexiletine concentrations were determined on the study day. $\mathrm{C}_{\max }$ and estimated $\mathrm{AUC}_{0-6}$ of mexiletine were $0.21 \pm 0.04 \mu \mathrm{g} / \mathrm{mL}$ and $0.86 \pm 0.19 \mu \mathrm{g} \bullet \mathrm{h} / \mathrm{mL}$, respectively (Table 1$)$.

\section{Change in blood pressure and $\mathrm{HR}$}

Changes in blood pressures and HR between the control and mexiletine phases were compared (Fig. 2 and Table 3). SBP and DBP in the mexiletine phase were significantly lower than those in control, respectively. Both pharmacodynamic markers for mexiletine phase were significantly lower than control $(P<0.05)$ (Table 3$)$. Reduction in SBP $(-10$ \pm 8 vs. $-24 \pm 7 \mathrm{mmHg})$ and $\mathrm{DBP}(-10 \pm 7$ vs. $-18 \pm 8 \mathrm{mmHg})$ after tizanidine administration were also significantly enhanced by coadministration of mexiletine $(P<0.01)$. Three subjects had drowsiness in the control phase and 10 subjects had drowsiness, dry mouth and/or dizziness 1-3 h after tizanidine administration in the mexiletine phase.

\section{Clinical survey from patients}

Of the 1,563 patients treated with tizanidine, 15 patients (male/female: 9/6; $60 \pm 13$ y; 
$61.2 \pm 12.8 \mathrm{~kg} ; 8$ non-smokers, 6 smokers and 1 unknown) had been coadministered mexiletine. Their daily doses of tizanidine and mexiletine, and the coadministration periods were $46.2(27.8-77.3) \mu \mathrm{g} / \mathrm{kg}$ and $3.9(1.8-6.0) \mathrm{mg} / \mathrm{kg}$, and 40 (1-791) days, respectively. SBP and DBP in mexiletine coadministration, which were recorded 2-6 h after tizanidine administration in 7 patients, were lower compared to those without coadministration (without vs. with: $125 \pm 16 / 82 \pm 16$ vs. $116 \pm 13 / 70 \pm 15 \mathrm{mmHg}$, $P<0.02)$. Reductions in SBP and DBP were $-9 \pm 6$ and $-12 \pm 7 \mathrm{mmHg}$, respectively. No patients received other medications known to inhibit tizanidine metabolism. The dosing schedule of drugs affecting blood pressure remained unchanged during the assessment period. Adverse effects caused by tizanidine were observed in 4 patients (drowsiness, 3; dry mouth, 1) who received $2.84 .2 \mathrm{mg} / \mathrm{kg}$ mexiletine daily and $2778.3 \mu \mathrm{gg} / \mathrm{kg}$ tizanidine daily. One patient discontinued combination therapy immediately after mexiletine was introduced because of drowsiness.

\section{DISCUSSION}

Present results suggested that combined use of mexiletine and tizanidine produced drug interaction in terms of enhancing the adverse effects of tizanidine. Coadministration of mexiletine $\left(2.2 \mathrm{mg} / \mathrm{kg}\right.$ daily) increased $\mathrm{C}_{\max }$, AUC and $\mathrm{t}_{1 / 2}$ of 
tizanidine to 3.1-, 3.6- and 1.4-fold, respectively, as compared to tizanidine alone in healthy subjects (Fig. 1, Table 2). These changes could be due to competitive inhibition of mexiletine on tizanidine metabolism via CYP1A2 because the metabolic clearance of tizanidine was decreased to one-third by mexiletine coadministration (Table 2). The pharmacodynamics of tizanidine, reduction in blood pressures, was also enhanced by mexiletine coadministration (Table 3). Because mexiletine did not alter SBP, DBP and HR at the blood concentration of $0.32-1.44 \mu \mathrm{g} / \mathrm{mL}$, which was higher than present study, blood pressure reduction in mexiletine phase was caused by tizanidine. ${ }^{21}$

Since the smoking, a possible factor for CYP1A2 induction, has been known to affect pharmacokinetics of tizanidine, ${ }^{16}$ it was speculated that the magnitude for drug interaction of tizanidine and mexililetine might differ between smokers and non-smokers. Our subgroup analyses, however, revealed that the effects of mexiletine coadministraion on tizanidine pharmacokinetics in smokers were similar to those in non-smokers (data not shown). No significant difference in any pharmacokinetic parameters of tizanidine in terms of smoking status also did not support the abovementioned speculation, although the power was not sufficient to reach the conclusion. We, therefore, analysed the present data without mention of smoking status.

The change in pharmacokinetics of tizanidine could be more remarkable in the 
practical use of mexiletine, because mexiletine was administered at a dose of 3.9 (1.8-6.0) $\mathrm{mg} / \mathrm{kg}$ daily for 40 days $(\mathbf{1 9 1 )}$, which is higher than that in the present study for healthy subjects $(2.2 \mathrm{mg} / \mathrm{kg}$ daily) (Table 1$)$. Because this dosage regimen produces higher steady state plasma mexiletine $(0.19-0.55 \mu \mathrm{g} / \mathrm{mL})$ as compared to the present $\mathrm{C}_{\max }(0.21 \mu \mathrm{g} / \mathrm{mL})$, CYP1A2 activity could be strongly impaired. In this situation, it is considered that the adverse effects of tizanidine readily occur even at the standard dose. Patients who suffered the adverse effects of tizanidine such as drowsiness and dry mouth had received $2482 \mathrm{mg} / \mathrm{kg}$ mexiletine daily and $27.787 .3 \mu \mathrm{g} / \mathrm{kg}$ tizanidine daily, which seem to be higher than the doses in the present pharmacokinetic study (2.2 mg/kg daily and $29.4 \mu \mathrm{g} / \mathrm{kg}$ daily, respectively). The blood pressure reduction was also observed during coadministration of tizanidine and mexiletine in 6 of the 7 patients whose blood pressures were assessed in the practical use of the combination therapy.

Previous 4 studies have documented that the pharmacokinetics of tizanidine was altered by coadministration of several CYP1A2 inhibitors (rofecoxib, ciprofloxacin, fluvoxamin and oral contraceptives) in healthy subjects, whereby elevation of blood tizanidine concentrations and reduction in blood pressure and HR were observed simultaneously. ${ }^{1,4,5,22}$ Effects of these CYP1A2 inhibitors on increase in plasma 
tizanidine concentration $\left(\mathrm{AUC}_{\text {inhibitor }} / \mathrm{AUC}_{\mathrm{control}}\right.$ ratio) were different: fluvoxamine (100 mg for 4 days), 33; ciprofloxacin (1000 mg for 3 days), 10; rofecoxib (25 mg for 4 days), 13.6 and oral contraceptives users, $3.9 .{ }^{22}$ Comparing these data, the magnitude of the effects of mexiletine on the pharmacokinetics of tizanidine is comparable with oral contraceptives because the $\mathrm{AUC}_{\text {inhibitor }} / \mathrm{AUC}_{\text {control }}$ ratio in the present study was 3.6.

The clinical effect of these tizanidine-related drug interactions depends on how frequent they are prescribed simultaneously. Because fluvoxamine and ciprofloxacin are now contraindicated for patients receiving tizanidine in Japan, they would no longer be simultaneously prescribed with tizanidine. Rofecoxib is not commercially available in Japan. Coadministration of oral contraceptives and mexiletine should be carefully monitored for tizanidine users in the future prospective study. Mexiletine was recently approved for treating painful neuropathies. ${ }^{23}$ Current survey of prescriptions in our hospital revealed that the chance for simultaneous use of mexiletine and tizanidine was gradually increasing (data not shown), even though caution was advised in package inserts for tizanidine products.

In conclusion, mexiletine coadministration altered the pharmacokinetics of tizanidine in a similar magnitude to that observed with oral contraceptives, which are known potent CYP1A2 inhibitors. Because mexiletine and tizanidine could be coadministered 
frequently for painful neuropathies, physicians, pharmacists and other medical staff should consider the clinical benefit/risk evaluation of this combination therapy for patients with neuropathy. 


\section{REFERENCES}

1. Backman JT, Karjalainen MJ, Neuvonen M, Laitila J, Neuvonen PJ. Rofecoxib is a potent inhibitor of cytochrome P450 1A2: studies with tizanidine and caffeine in healthy subjects. Br J Clin Pharmacol 2006; 62: 345-357.

2. Karjalainen MJ, Neuvonen PJ, Backman JT. Celecoxib is a CYP1A2 inhibitor in vitro but not in vivo. Eur J Clin Pharmacol 2008; 64: 511-519.

3. Karjalainen MJ, Neuvonen PJ, Backman JT. Tolfenamic acid is a potent CYP1A2 inhibitor in vitro but does not interact in vivo: correction for protein binding is needed for data interpretation. Eur J Clin Pharmacol 2007; 63: 829-836.

4. Granfors MT, Backman JT, Neuvonen M, Neuvonen PJ. Ciprofloxacin greatly increases concentrations and hypotensive effect of tizanidine by inhibiting its cytochrome P450 1A2-mediated presystemic metabolism. Clin Pharmacol Ther 2004; 76: 598-606.

5. Granfors MT, Backman JT, Neuvonen M, Ahonen J, Neuvonen PJ. Fluvoxamine drastically increases concentrations and effects of tizanidine: a potentially hazardous interaction. Clin Pharmacol Ther 2004; 75: 331-341.

6. Momo K, Doki K, Hosono H, Homma M, Kohda Y. Drug interaction of tizanidine and fluvoxamine. Clin Pharmacol Ther 2004; 76: 509-510.

7. Momo K, Homma M, Kohda Y et al. Drug interaction of tizanidine and ciprofloxacin: 
case report. Clin Pharmacol Ther 2006; 80: 717-719.

8. Package inserts of Ternelin ${ }^{\circledR}$, http://www.novartis.co.jp/product/ten/pi/pi_ten.pdf (last accessed: 19 November 2008)

9. Konishi H, Morita K, Minouchi T, Yamaji A. Preferential inhibition of CYP1A enzymes in hepatic microsomes by mexiletine. Eur J Drug Metab Pharmacokinet 1999; 24: 149-153.

10. Kobayashi K, Nakajima M, Chiba K et al. Inhibitory effects of antiarrhythmic drugs on phenacetin O-deethylation catalysed by human CYP1A2. Br J Clin Pharmacol 1998; 45: 361-368.

11. Kendall JD, Chrymko MM, Cooper BE. Theophylline-mexiletine interaction: a case report. Pharmacotherapy 1992; 12: 416-418.

12. Ellison MJ, Lyman DJ, San Miguel E. Threefold increase in theophylline serum concentration after addition of mexiletine. Am J Emerg Med 1992; 10: 506-508.

13. Hurwitz A, Vacek JL, Botteron GW et al. Mexiletine effects on theophylline disposition. Clin Pharmacol Ther 1991; 50: 299-307.

14. Stoysich AM, Mohiuddin SM, Destache CJ, Nipper HC, Hilleman DE. Influence of mexiletine on the pharmacokinetics of theophylline in healthy volunteers. J Clin Pharmacol 1991; 31: 354-357.

15. Ueno K, Miyai K, Seki T, Kawaguchi Y. Interaction between theophylline and 
mexiletine. DICP 1990; 24: 471-472.

16. Backman JT, Schröder MT, Neuvonen PJ. Effects of gender and moderate smoking on the pharmacokinetics and effects of the CYP1A2 substrate tizanidine. Eur J Clin Pharmacol 2008; 64: 17-24.

17. Nirogi RV, Kandikere VN, Shukla M, Mudigonda K, Maurya S. Quantification of tizanidine in human plasma by liquid chromatography coupled to tandem mass spectrometry. Rapid Commun Mass Spectrom 2006; 20: 2286-2292.

18. Mastropaolo W, Holmes DR, Osborn MJ, Rooke J, Moyer TP. Improved liquid-chromatographic determination of mexiletine, an antiarrhythmic drug, in plasma. Clin Chem 1984; 30: 319-322.

19. Higuchi S, Aoyama T, Horioka M. PEDA: a microcomputer program for parameter estimation and dosage adjustment in clinical practice. J Pharmacobiodyn 1987; 10: 703-718.

20. Chen X, Wang L, Zhi L et al. The G-113A polymorphism in CYP1A2 affects the caffeine metabolic ratio in a Chinese population. Clin Pharmacol Ther 2005; 78: 249-259.

21. Katagiri M, Yamada $M$, Imai $K$ et al. Effectiveness of mexiletine in senile hypertensives with repetitive ventricular arrhythmias and serum mexiletine concentration measured with HPLC. Jpn J Clin Pharmacol Ther 1988; 19: 423-430.

22. Granfors MT, Backman JT, Laitila J, Neuvonen PJ. Oral contraceptives containing 
ethinyl estradiol and gestodene markedly increase plasma concentrations and effects of tizanidine by inhibiting cytochrome P450 1A2. Clin Pharmacol Ther 2005; 78: $400-411$.

23. Package inserts of Mexitil ${ }^{\circledR}$ Capsules, http://www.boehringer-ingelheim.co.jp/ products/ prescription/medicine_data/mx_cap/mx_cap_tenpu.pdf (last accessed: 15 January 2009). 


\section{FIGURE LEGENDS}

Fig. 1 Plasma concentrations (mean \pm SD) of tizanidine in healthy subjects after administration of tizanidine (2 mg) alone (open circles) and coadministration of tizanidine (2 mg) and mexiletine (solid circles).

Fig. 2 Blood pressure (mean \pm SD) in healthy subjects after administration of tizanidine (2 mg) alone (open circles) and coadministration of tizanidine (2 mg) and mexiletine (solid circles). 
Fig. 1

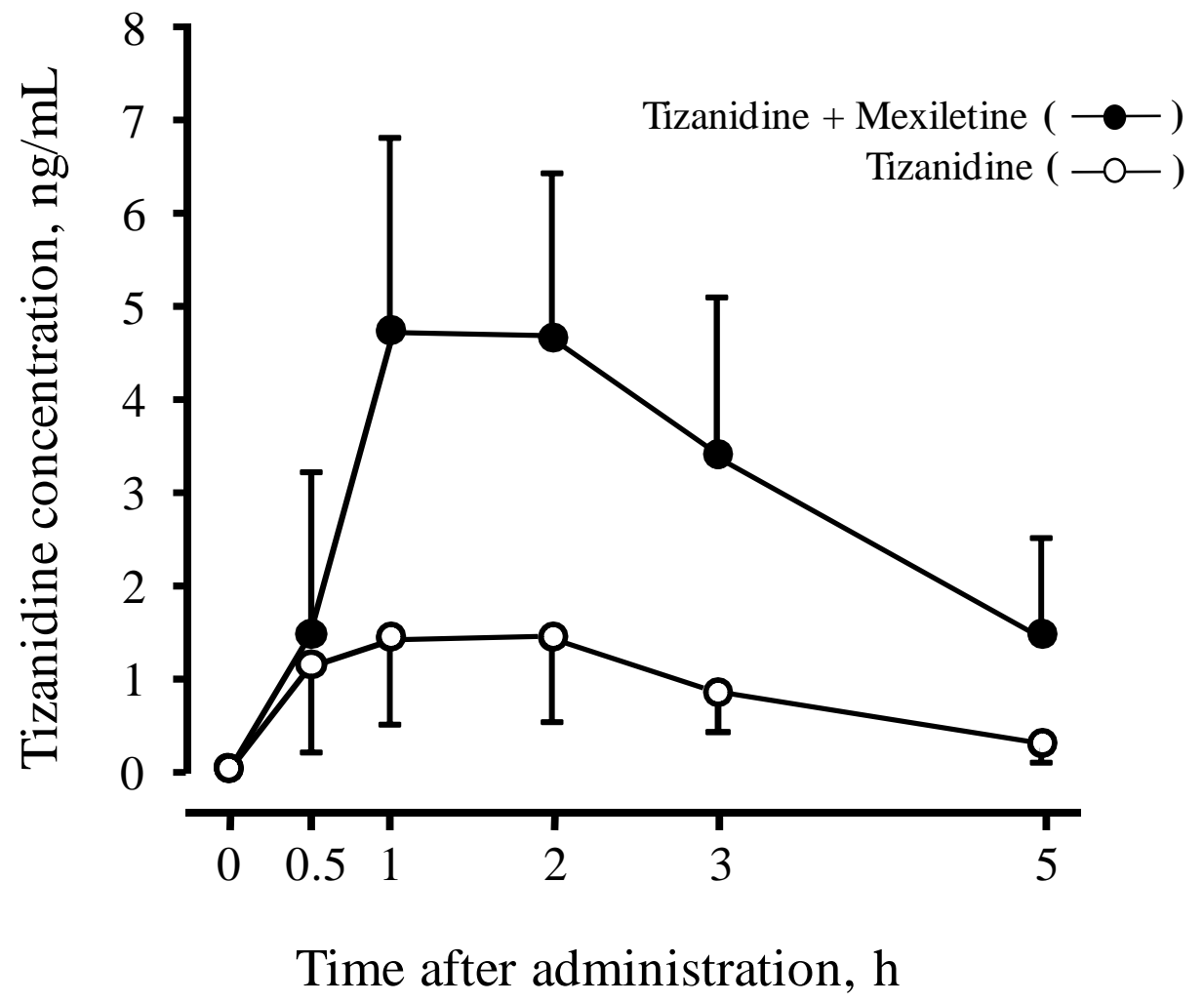


Fig. 2

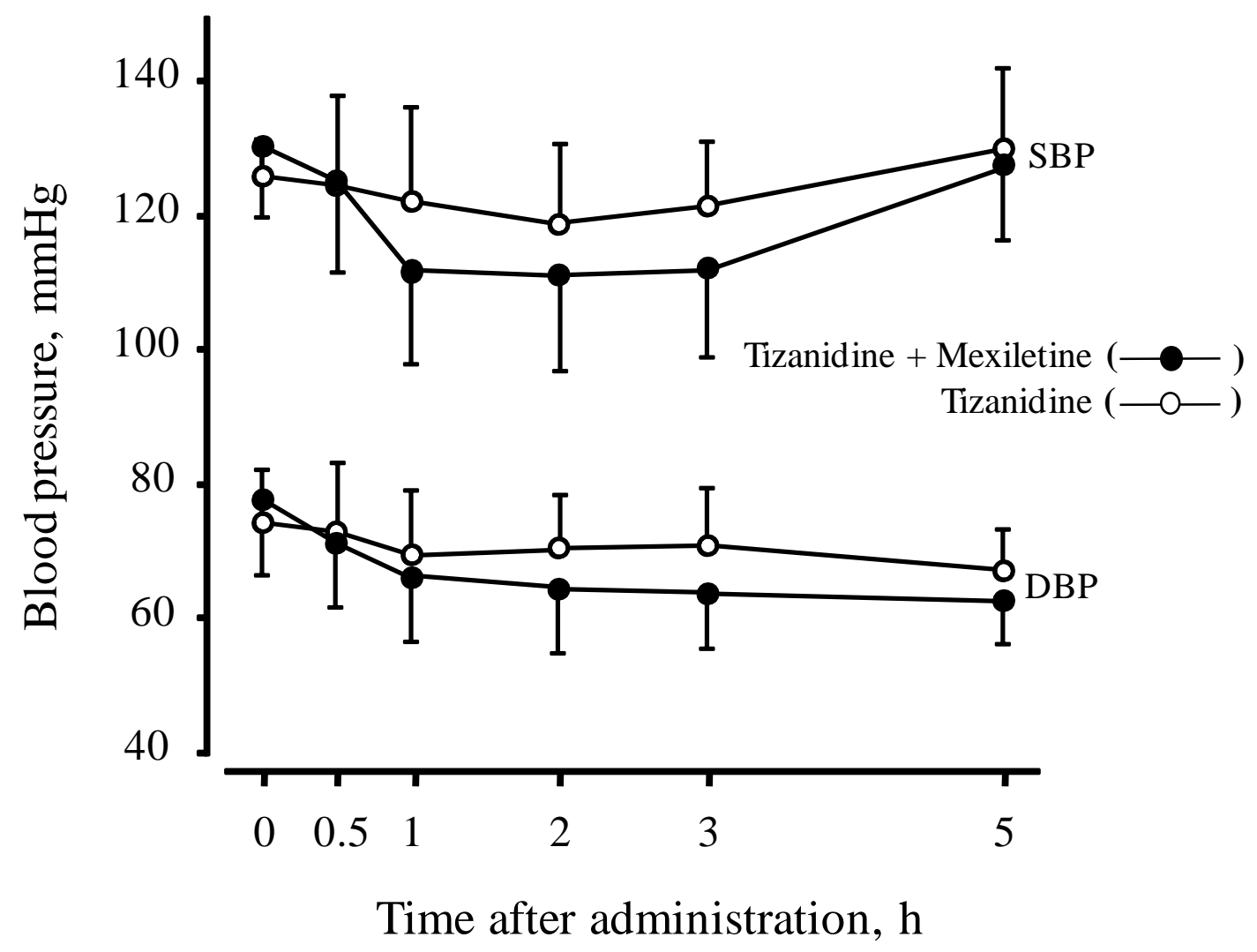


Table 1 The profile of healthy subjects

\begin{tabular}{lc}
\hline Number (Male/Female) & $12(12 / 0)$ \\
Smoker/Non-smoker & $5 / 7$ \\
Weight, kg & $69.9 \pm 12.1$ \\
Age, y & $29 \pm 5$ \\
Mexiletine dose, mg/kg/day & $2.2 \pm 0.4$ \\
Tizanidine dose, $\mu \mathrm{g} / \mathrm{kg}$ & $29.4 \pm 5.0$ \\
Mexiletine pharmacokinetic parameters & \\
$\mathrm{C}_{\mathrm{max}}, \mu \mathrm{g} / \mathrm{mL}$ & $0.21 \pm 0.04$ \\
$\mathrm{AUC}_{0-6}, \mu \mathrm{g} \cdot \mathrm{h} / \mathrm{mL}$ & $0.86 \pm 0.19$ \\
\hline
\end{tabular}

Data are represented as mean $\pm \mathrm{SD}$.

$\mathrm{C}_{\max }$ : Peak concentration, $\mathrm{AUC}_{0-6}$ : Area under the plasma concentration

versus time curve from 0 to $6 \mathrm{~h}$ after administration. 
Table 2 Pharmacokinetic parameters of tizanidine in healthy subjects after administration of tizanidine (2 mg) alone and coadministration of tizanidine $(2 \mathrm{mg}$ ) and mexiletine

\begin{tabular}{lllll}
\hline Variable & Tizanidine & Tizanidine + Mexiletine & $\begin{array}{l}\text { Times of control } \\
(95 \% \mathrm{CI})\end{array}$ & $P$ value \\
\hline $\mathrm{C}_{\max }, \mathrm{ng} / \mathrm{mL}$ & $1.8 \pm 0.8$ & $5.3 \pm 1.8$ & $3.1(2.6,3.6)$ & $<0.001$ \\
$\mathrm{t}_{\max }, \mathrm{h}$ & $1.2(0.5,2.0)$ & $1.5(1.0,2.0)$ & - & - \\
$\mathrm{AUC}_{0-5}, \mathrm{ng} \cdot \mathrm{h} / \mathrm{mL}$ & $4.5 \pm 2.2$ & $15.4 \pm 6.5$ & $3.6(2.9,4.3)$ & $<0.001$ \\
$\mathrm{t}_{1 / 2}, \mathrm{~h}$ & $1.3 \pm 0.2$ & $1.8 \pm 0.7$ & $1.4(1.2,1.6)$ & $<0.05$ \\
$\mathrm{CL} / \mathrm{F}, \mathrm{L} / \mathrm{h}$ & $462 \pm 209$ & $133 \pm 55$ & $0.31(0.23,0.39)$ & $<0.001$ \\
$\mathrm{CL}_{\mathrm{R}} / \mathrm{F}, \mathrm{L} / \mathrm{h}$ & $2.6 \pm 1.1$ & $2.0 \pm 1.1$ & $0.86(0.56,1.17)$ & $\mathrm{NS}$ \\
$\mathrm{CL}_{\mathrm{NR}} / \mathrm{F}, \mathrm{L} / \mathrm{h}$ & $460 \pm 209$ & $131 \pm 55$ & $0.31(0.23,0.39)$ & $<0.001$ \\
\hline
\end{tabular}

Data are represented as mean \pm SD or median (range).

CI: Confidence interval, $\mathrm{C}_{\max }$ : Peak concentration, $\mathrm{t}_{\max }$ : Time for $\mathrm{C}_{\max }, \mathrm{AUC}_{0-5}$ : Area under the plasma concentration versus time curve from 0 to $5 \mathrm{~h}$ after administration, $\mathrm{t}_{1 / 2}$ : Half-life, CL/F: Apparent oral clearance, $\mathrm{CL}_{\mathrm{R}} / \mathrm{F}$ : Renal clearance, $\mathrm{CL}_{\mathrm{NR}} / \mathrm{F}$ : Non-renal clearance, NS: Not significant. 
Table 3 Changes in cardiovascular pharmacodynamic parameters in healthy subjects after administration of tizanidine alone and coadministration of tizanidine and mexiletine

\begin{tabular}{|c|c|c|c|c|}
\hline Variable & Tizanidine & Tizanidine+Mexiletine & $\begin{array}{l}\text { Difference between } \\
\text { phases ( } 95 \% \text { CI) }\end{array}$ & $P$ value \\
\hline \multicolumn{5}{|l|}{ Systolic blood pressure } \\
\hline Baseline, mmHg & $126 \pm 6$ & $130 \pm 10$ & $4(-1,10)$ & NS \\
\hline Minimum, mmHg & $116 \pm 12$ & $107 \pm 12$ & $-9(-15,-3)$ & $<0.01$ \\
\hline $\mathrm{AUC}_{0-5}, \mathrm{mmHg} \bullet \mathrm{h}$ & $616 \pm 50$ & $586 \pm 56$ & $-30(-55,-5)$ & $<0.05$ \\
\hline \multicolumn{5}{|l|}{ Diastolic blood pressure } \\
\hline Baseline, mmHg & $75 \pm 8$ & $78 \pm 11$ & $3(-3,10)$ & NS \\
\hline Minimum, mmHg & $64 \pm 6$ & $60 \pm 7$ & $-5(-9,-1)$ & $<0.05$ \\
\hline $\mathrm{AUC}_{0-5}, \mathrm{mmHg} \bullet \mathrm{h}$ & $352 \pm 37$ & $328 \pm 37$ & $-24(-46,-2)$ & $<0.05$ \\
\hline \multicolumn{5}{|l|}{ Heart rate } \\
\hline Baseline, beats/min & $78 \pm 13$ & $76 \pm 15$ & $-2(-6,2)$ & NS \\
\hline Minimum, beats/min & $67 \pm 8$ & $63 \pm 8$ & $-4(-7,0)$ & $<0.05$ \\
\hline $\mathrm{AUC}_{0-5}$, beats $/ \mathrm{min} \cdot \mathrm{h}$ & $365 \pm 44$ & $351 \pm 45$ & $-14(-29,0)$ & $<0.05$ \\
\hline
\end{tabular}

Data are represented as mean $\pm \mathrm{SD}$.

CI: Confidence interval, $\mathrm{AUC}_{0-5}$ : Area under the blood pressure or heart rate versus time curve from 0 to $5 \mathrm{~h}$ after administration, NS: Not significant. 